 \\ A bi-static sodar for precision wind profiling in complex terrain
}

Sabine von Hünerbein

\section{Torben Mikkelsen}

Wind Energy Division, Risø National Laboratory for Sustainable Energy, Technical University of Denmark, Denmark

\footnotetext{
${ }^{1}$ Corresponding author address: Stuart Bradley, Physics Department, University of Auckland, Private Bag 92019, Auckland, New Zealand

E-mail: s.bradley@auckland.ac.nz
} 
21 A new ground-based wind profiling technology, a scanned bistatic sodar, is described.

22 The motivation for this design is to obtain a 'mast-like' wind vector profile in a single

23 atmospheric column extending from the ground to heights of more than $200 \mathrm{~m}$. The need

24 for this columnar profiling arises from difficulties experienced by all existing lidars and

25 sodars in the presence of non-horizontally-uniform wind fields, such as found generically

26 in complex terrain. Other advantages are described, including improved signal strength

27 from turbulent velocity fluctuations, improved data availability in neutral atmospheric

28 temperature profiles, improved rejection of rain echoes, and improved rejection of echoes

29 from fixed (non-atmospheric) objects. Initial brief field tests indicate that the scattered

30 intensity profile agrees with theoretical expectations, and bistatic sodar winds are

31 consistent with winds from standard mast-mounted instruments. 


\section{1. Introduction}

35 In the last few years the use of surface-based remote sensing for wind energy has come to

36 be the preferred method of obtaining wind profiles in the vicinity of large turbines

37 (Upwind, 2011). The useful instruments comprise two types: lidars, which use laser light

38 scattered from naturally occurring atmospheric particulates; and sodars, which use

39 audible sound scattered from atmospheric turbulence (Emeis, 2010). Wind components

40 are sensed through the Doppler frequency shift of the light or sound caused by the

41 movement of the target particles or turbulence in the radiated volume above the

42 instrument. Although continuous systems exist, such as the ZephIR lidar (Natural Power,

43 2010), nearly all lidars and sodars are pulsed, and the position in the atmosphere from

44 which the scattering occurs is determined by time-of-flight of the returning signal. Both

45 the optical and the acoustic instruments are faced with the challenge of low received

46 signal levels compared with background noise.

47 All commercial versions of lidars and sodars are 'mono-static', by which is meant

48 that the transmitter and receiver are co-located, and energy from the scattering volume is

49 scattered through $180^{\circ}$. This has the advantage of compactness, and the instruments are

50 more readily deployed in the field because the single instrument package is self-

51 contained. However, Doppler shift from a moving target requires that there be a

52 component of the motion either in the transmitter-target line or in the target-receiver line.

53 This means that, to sense three Cartesian coordinate wind components, at least three 
54 beams of light or sound have to be transmitted upward and at least two of these must be

55 non-vertical.

$56 \quad$ For a sound beam transmitted in the direction $\mathbf{T}$ and scattered energy received

57 from direction $\mathbf{R}$, the measured Doppler shift can be written in scaled form as

$$
\mu=-\frac{c}{2} \frac{\Delta f}{f_{T}}=\frac{1}{2}\left(\frac{\mathbf{T}}{|\mathbf{T}|}+\frac{\mathbf{R}}{|\mathbf{R}|}\right) \bullet \mathbf{V}
$$

60 where $c$ is the speed of sound, $\Delta f$ the Doppler shift, $f_{T}$ the frequency of the transmitted

61 sound, and $\mathbf{V}=(u, v, w)$ the wind velocity vector (Bradley, 2007). In the mono-static

62 case, $\mathbf{T}=\mathbf{R}$, and three measurements would give

$$
\mu_{b}=u_{b} \sin \theta_{b} \cos \phi_{b}+v_{b} \sin \theta_{b} \sin \phi_{b}+w_{b} \cos \theta_{b} \quad b=1,2,3
$$

65 where $\theta_{b}$ and $\phi_{b}$ are the zenith and azimuth angles of the $b^{\text {th }}$ beam direction. If $u_{b}=u, v_{b}=$

$66 v$, and $w_{b}=w$ for $b=1,2,3$, then the equations can be solved for the Cartesian wind

67 components $u, v$, and $w$.

\section{a. Wind estimation errors in complex terrain}

69 Solving (2) for $(u, v, w)$ requires the assumption of horizontal homogeneity of the wind

70 field, which is probably sufficiently valid above flat terrain, but seldom valid over

71 complex terrain. The $u$ components $u_{1}, u_{2}$, and $u_{3}$, for example, are in general different

72 because they are the values of the $u$ component in three different volumes. Generally it is

73 the components directly above the instrument which are required, since this gives 'mast-

74 like' wind profiles. Bradley (2008) has developed a potential flow model for estimating

75 remote sensing errors over a bell-shaped hill. The fractional error in estimating the wind

76 speed for a 3-beam sodar sited on the crest of the hill, with beam 1 facing downwind, is 


$$
\frac{\hat{V}}{V_{0}}-1 \approx-2 \eta^{2} \frac{z}{H} \approx-5 G_{\max }^{2} \frac{z}{H}
$$

79 where $z$ is the height of the sensing volume above the hill crest, $H$ is the hill height, $\eta$ is

80 the ratio of hill height to hill half-width at half-height, and $G_{\max }$ is the maximum gradient

81 of the bell-shaped hill. The fractional error is negative because the maximum speed is

82 directly above the instrument in this case, and the beam directed in the direction of the

83 flow underestimates. So for a hill of maximum gradient 0.1 , and with $z=H$, a 5\% error in

84 wind estimation is predicted. This is comparable to the error measured in practice in

85 complex terrain (Behrens et al., 2011, Bradley et al., 2011), and is unacceptably high for

86 wind energy applications. Note that this error is generic across all sodars and lidars, and

87 is insensitive to the beam zenith angle $\theta$.

$88 \quad$ Bingol et al. (2009) have proposed a correction method using a flow model

89 (Wasp). However, the reason for doing the in situ remote sensing measurements is

90 because the available flow models are considered insufficiently reliable in complex

91 terrain. This raises the question of whether correcting inaccurate measurements using

92 inaccurate models is a useful approach.

93 One approach to the distributed sensing volume problem is to expand the wind

94 component variations in the horizontal using Taylor series (Bradley et al., 2011). For

95 example, the $u$ component expands as

$$
u(\mathbf{T})=u(\mathbf{Z})+\left.[(\mathbf{T}-\mathbf{Z}) \bullet \nabla] u\right|_{\mathbf{Z}}+\ldots
$$

97 The correct component above the instrument is $u(\mathbf{Z})$, and the error term contains

98 the vector distance $\mathbf{T}-\mathbf{Z}$ horizontally between the sample volume and the point above the

99 instrument. Note that this expansion does not include $\mathbf{R}$. What this means is that, 
100 provided transmission is vertical (i.e. $\mathbf{T}=\mathbf{Z}$ ), there are no corrections due to horizontal

101 wind shear. But from (1), a mono-static instrument with $\mathbf{T}=\mathbf{Z}$ can only sense $\mathbf{Z} \bullet \mathbf{V}=w$.

102 The main rationale for the work described in this publication is to describe a remote

103 sensing system in which $\mathbf{T}=\mathbf{Z}$ but $\mathbf{R} \neq \mathbf{T}$. Systems in which $\mathbf{R} \neq \mathbf{T}$ are called 'bi-static',

104 and necessarily have separated transmitter and receivers, as shown in Figure 1.

105 b. Previous bi-static sodar designs

106 The Doppler shift and scattering cross section for bi-static sodars were analysed by

107 Thompson and Coulter (1974) and by Wesely (1976). Early experiments with bi-static

108 sodars are described by Coulter and Underwood (1980) and Underwood (1981) for the

109 Risø-78 experiment. For this experiment there were two bi-static sodars, as shown in

110 Figure 2. Bi-static system (a) transmitted at $1 \mathrm{kHz}$, and system (b) at $1.6 \mathrm{kHz}$. Both

111 systems operated in 'staring', or non-scanning, mode (the tilted beams had a fixed zenith

112 angle of $60^{\circ}$ ), but the overlap between the vertical beam and the tilted beams was from

113 about 90 to $200 \mathrm{~m}$ height, allowing for profiling over this height range with pulsed

114 transmission. Both systems were pulsed, defining an instantaneous sensing volume of

115 depth of about $17 \mathrm{~m}$. Comparisons with tower measurements $260 \mathrm{~m}$ distant are shown in

116 Figure 3. Although 30 minute averages were used, the uncertainties in the bi-static wind

117 measurements are rather large. Values of the structure function parameter for turbulence

118 velocity fluctuations, $C_{V}^{2}$ were also measured at a height of $130 \mathrm{~m}$. The azimuth and

119 elevation angles could be changed manually but this took around 4 minutes.

120 Mastrantonio et al. (1986) also presented some preliminary results of use of a

121 staring mode bi-static sodar which could be used simultaneously with a 3-axis monostatic

122 sodar, and Mathews et al. (1986) explored refractive acoustic path bending effects for bi- 
123 static sodars. Moulsley and Cole (1993) extended the earlier analyses to give a general

124 radar equation for bi-static sodars. Zinichev et al. (1997) have described a very large bi-

125 static system having transmitter-receiver separations of $400 \mathrm{~m}$.

126 Mikkelsen et al.(2007) have described 'Heimdall', a continuous-transmission

127 staring-mode bi-static sodar design. This operated with vertical transmission at $4 \mathrm{kHz}$ and

128 a single receiver beam of $45^{\circ}$ zenith angle at a separation distance of $60 \mathrm{~m}$. The combined

129 temperature structure function parameter $C_{T}^{2}$ and velocity structure function $C_{V}^{2}$

130 measurements agreed with mast measurements to within an order of magnitude, which is

131 reasonable, given various system uncertainties. It was noted that only $25 \%$ of the

132 received scattered energy was expected to be from temperature fluctuations.

133 Figure 4 shows a spectrum from the Heimdall bi-static sodar. The direct signal

134 from the transmitter to the receiver is obvious in the sharp spectral peak at $3960 \mathrm{~Hz}$. The

135 remainder of the spectral hump is comprised of two broad bell-shaped spectral peaks.

136 The broader spectral peak to the left is due to the vertically transmitted pulse. Note that it

137 is much broader than the direct signal spectrum because of the wide range of scattering

138 angles for this continuous system. There is also a second broader peak, partly underlying

139 the direct signal peak and slightly to its right. This is due to a diffraction side lobe from

140 the dish antenna used. Given that $f_{T}=3960 \mathrm{~Hz}$, and the peak at the left is at $3920 \mathrm{~Hz}$ (for

$141 \theta=0), u / c=(40 / 3960) R / D$, where $R=\left(D^{2}+z^{2}\right)^{1 / 2}$ is the distance from receiver to sensing

142 volume, and $D$ is the distance from the receiver to the point below the sensing volume.

143 The half-width of the left-hand spectral peak is about $50 \mathrm{~Hz}$, so the range of scattering

144 angles, expressed as $\Delta \theta$, is 


$$
\Delta \theta=\frac{50}{3960} \frac{1}{\frac{u}{c}\left(1+\frac{z}{R}\right)}=\frac{50}{3960} \frac{D}{\frac{40}{3960}(R+z)}=1.25 \frac{D}{R+z}
$$

146 Here $D=z$, so $\Delta \theta= \pm 30^{\circ}$, which emphasizes the need for bi-static SODARs to be

147 pulsed systems. The broad peak at the right, at $3970 \mathrm{~Hz}$, will be from a side-lobe at about

$14827^{\circ}$ from the vertical. Side lobes at such angles readily exist since they will generally be

149 within the angular pass region of acoustic baffles. For mono-static SODARs such a side

150 lobe would be unlikely to cause problems, but in the case of this bi-static system it is

151 significant.

152 Very recently AQS (2010) have announced a commercial 'common volume'

153 configuration comprising three interconnected sodars each having tilted beams which

154 intersect at a common volume in staring mode. A typical configuration is quoted as

155 having the three beams all with zenith angle $\theta=15^{\circ}$, the common volume at height $z=$

$156100 \mathrm{~m}$, and the three sodar units each separated from the point on the ground beneath the

157 sensed volume by a distance of $D=26 \mathrm{~m}$. The system is pulsed, giving better definition of

158 the sensed volume. Winds can be obtained only from a single height. Previous bi-static

159 designs discussed above, and the design by Shamanaev(2003), all used fixed angle

160 antennas, with the limitation of a rather confined height range.

161 From these examples of previous work it is clear that bi-static sodar systems do

162 give wind profiles, but that (1) they should allow for a non-staring (i.e. scanned) mode, or

163 a multiple fan-beam staring mode, so as to give a broad height range, and (2) they should

164 be pulsed so that problems with direct and diffracted beam reception are avoided, and so

165 that the height range of the sensed volume is not so extensive. The design described

166 below accommodates to these requirements. 


\section{2. Bi-static sodar design principles}

168 Both the Doppler shift and the received amplitude are different for a bi-static system

169 compared with a mono-static sodar. While any configuration of three beams could be

170 used (such as the AQS configuration), if the atmosphere is to be scanned in a column, it

171 is more convenient to have one beam pointing vertically, since then only two beams need

172 be scanned. We will concentrate discussion on a single vertical transmission beam and

173 two tilted receiving beams, with the two planes defined by each tilted beam and the

174 vertical being orthogonal, as in Figure 5.

175 a. Signal Amplitude

176 Scattered acoustic power $P_{R}$ is given by:

$$
P_{R} \propto \frac{\sin ^{2} \beta}{(1+\sin \beta)^{11 / 6}} \frac{e^{-\alpha r}}{r^{2}}\left[C_{T}^{2}+3.66(1-\sin \beta) \frac{T^{2}}{c^{2}} C_{V}^{2}\right]
$$

178 where $r=z+\left(D^{2}+z^{2}\right)^{1 / 2}$ is the total sound path distance, $c$ is the speed of sound in air, $T$

179 the absolute air temperature, $\alpha$ the absorption coefficient, $C_{V}^{2}$ and $C_{T}^{2}$ are turbulent

180 structure function parameters, and $\beta=\tan ^{-1}(z / D)$ the elevation angle from the receiver to

181 the sensing volume (Bradley, 2007). Bi-static SODARs have greater sensitivity than

182 mono-static SODARs because of the extra contribution from $C_{V}^{2}$, especially in neutral

183 conditions when $C_{T}^{2}$ vanishes.

184 b. Sensitivity to scattering from rain

185 Acoustic scattering from rain drops for typical SODAR wavelengths is in the Rayleigh

186 regime, and has an angular dependence of $(\sin \beta-2 / 3)^{2}$, as discussed by Bradley and 
187 Webb (2002). This has a minimum at $\sin \beta=2 / 3$ or $\beta=42^{\circ}$, whereas from Equation (6),

188 the scattering from velocity fluctuations peaks at $\beta=35^{\circ}$. This means that, for much of the

189 bi-static profile, the angular scattering patterns of turbulence and rain favour the

190 scattering from turbulence.

191 The scattered energy amplitudes from temperature and velocity fluctuations are

192 shown in Figure 6 for $D=30 \mathrm{~m}$ and for $D=50 \mathrm{~m}$, together with the scattering pattern from

193 rain.

c. Doppler winds

195 From (1), the bi-static equivalent of (2) is

196

$$
\begin{aligned}
& \mu_{b}=\frac{u}{2} \cos \beta \cos \phi_{i}+\frac{v}{2} \cos \beta \sin \phi_{i}+\frac{w}{2}(1+\sin \beta) \quad b=1,2 \\
& \mu_{3}=w
\end{aligned}
$$

with the solution, for $\phi_{1}=\phi_{2}-90^{\circ}=\phi$,

$$
\begin{aligned}
& u=\left[2\left(\mu_{1} \cos \phi-\mu_{2} \sin \phi\right)-(1+\sin \beta) \mu_{3}(\cos \phi-\sin \phi)\right] / \cos \beta \\
& v=\left[2\left(\mu_{1} \sin \phi+\mu_{2} \cos \phi\right)-(1+\sin \beta) \mu_{3}(\cos \phi+\sin \phi)\right] / \cos \beta \\
& w=\mu_{3}
\end{aligned}
$$

For example, if $\phi=0$, the coefficient of $u$ in $\mu_{1}$, which is proportional to the Doppler shift in beam 1 from the $u$ component, is greater than the corresponding monostatic Doppler shift up to the height of $83 \mathrm{~m}$ if the bi-static spacing $D=50 \mathrm{~m}$, and the mono-static beam zenith angle is $\theta=15^{\circ}$.

The Doppler contribution from $w$ in beams 1 and 2 is always larger than the mono-static case. This increased Doppler helps discriminate against echoes from fixed objects around the sodar. For example, assume a hard reflecting surface is at a range of $20 \mathrm{~m}$ and the atmospheric scattered signal is of the same amplitude as that from the fixed 
209 surface. For a horizontal wind speed component of $2 \mathrm{~m} \mathrm{~s}^{-1}$ in the plane of a beam, and

210 with a pulse duration of $0.1 \mathrm{~s}$, a transmitted frequency of $4500 \mathrm{~Hz}$, a mono-static beam

211 zenith angle of $15^{\circ}$, and a bi-static baseline of $D=50 \mathrm{~m}$, Figure 7 shows the

212 corresponding Doppler spectra for a mono-static sodar and a bi-static sodar. The much

213 improved resolving power of the bi-static system is evident.

$214 \quad$ d. Scanning geometry

215 Sodars normally have a pulse duration of about $\tau=0.1 \mathrm{~s}$, corresponding to a height

216 resolution of $\Delta z=c \tau / 2=17 \mathrm{~m}$. In the case of a scanning, pulsed, bi-static design, the

217 pulse height will define the sensing volume height, but for maximum signal gain the

218 beam width of the scanned beam should not be so large that much of the sensitive beam

219 area is outside the pulsed volume. The antenna for typical sodars has a diameter $L$ of

220 between $0.5 \mathrm{~m}$ and $1.0 \mathrm{~m}$, and the width of the sodar beam, from peak to the first null, is

221 about

$$
\Delta \beta=\frac{2 \pi}{k L}=\frac{c}{L f_{T}}
$$

223 where $k$ is the acoustic wavenumber and $f_{T}$ is the transmitted frequency. For $f_{T}=4500 \mathrm{~Hz}$,

$224 \Delta \beta=2.7^{\circ}$ for $L=0.8 \mathrm{~m}$. At $80 \mathrm{~m}$ height, for example, the diameter of this beam would be

225 about $15 \mathrm{~m}$, or close to the typical height extent defined by the pulse duration. Figure 8

226 shows schematically how the sampling volume is defined by the product of three

227 Gaussian spatial functions: one for the transmitted beam, one for the received beam, and

228 one for the transmitted pulse. 
For example, if $f_{\mathrm{T}}=4500 \mathrm{~Hz}, L=0.8 \mathrm{~m}, D=50 \mathrm{~m}$, and a Gaussian pulse is used

230 having a temporal standard deviation of $0.02 \mathrm{~s}$, the sampling volumes at 30,50 and $80 \mathrm{~m}$

231 are as shown in Figure 9.

232 Given the above, a reasonable design starting point is to have the scanned

233 receiving arrays about $1 \mathrm{~m}$ in length. For a prototype bi-static receiver, we have used

234 Motorola KSN1005A super-horn tweeters as microphones. These have a diameter of $d=$

$2350.085 \mathrm{~m}$ and, because our multi-channel data loggers have 12 channels, we used $M=12$ of

236 these microphones in a linear array, giving a length $L=0.935 \mathrm{~m}$. In order to limit the

237 lateral extent of the sensitivity, we used a 12x3 array, with each row of three

238 microphones connected in parallel to a low-noise preamplifier. This gave a lateral half-

239 beamwidth of $12.7^{\circ}$.

240 The pointing direction of each microphone array is controlled by adding a

241 progressive phase shift $\Delta \varphi$ to each row down the length of the linear array of

242 microphones (Bradley, 2007). In order to obtain best sensitivity, each array is mounted on

243 a tripod and aimed at a height $z_{0}$, at an elevation angle of $\beta_{0}$.

244 The pointing elevation angle, $\beta_{g}$ to the centre of a range gate sampling volume at 245 height $z_{g}$, is

$$
\beta_{g}=\beta_{0}+\sin ^{-1} \frac{\Delta \varphi}{k d}
$$

$$
\frac{\Delta \varphi}{k d}=\frac{D}{\left(D^{2}+z_{0}^{2}\right)^{1 / 2}} \frac{z_{g}-z_{0}}{\left(D^{2}+z_{g}^{2}\right)^{1 / 2}}
$$

249 The voltage output $s_{m}\left(t_{i}\right)$ from microphone $m(m=1,2, \ldots, M)$ is recorded at times $t_{i}=$ $250 i \Delta t(i=1,2, \ldots, N)$ with time $t=0$ being the start of the transmission of the acoustic pulse. 
251 The first scattered sound from the air just above the transmitter arrives at the receiver 252 array at time $t_{0}=D / c$. Signals from a range gate at height $z_{g} \pm \Delta z_{g} / 2$ arrive between time

$\left.253\left[\left(z_{g}-\Delta z_{g} / 2\right)+\left\{D^{2}+\left(z_{g}-\Delta z_{g} / 2\right)^{2}\right)\right\}^{1 / 2}\right] / \mathrm{c}$ and $\left.\left[\left(z_{g}+\Delta z_{g} / 2\right)+\left\{D^{2}+\left(z_{g}+\Delta z_{g} / 2\right)^{2}\right)\right\}^{1 / 2}\right] / \mathrm{c}$ or, say, $i=i_{g}$,

$254 i_{g}+1, \ldots, i_{g}+\left(N_{g}-1\right)$. Within this time period, the phased array receiver needs to be staring

255 at this sensing volume, which is achieved by applying the correct incremental phase shift

256 across the array microphone elements.

257 All of this processing can be done after recording the whole time series $s_{m}\left(t_{i}\right)$.

258 Delays of any precision can be applied through Fourier transforms.

$$
\begin{aligned}
& S_{m g}^{\prime}(f)=\int_{t_{i_{g}}}^{t_{i_{g}+\left(N_{g}-1\right)}} s_{m}\left(t-m \Delta t_{g}\right) e^{j 2 \pi f t} d t \\
& =\left(e^{j m \frac{f}{f_{T}} \Delta \varphi_{g}}\right) \int_{t_{i_{g}}+m \Delta t_{g}}^{t_{i_{g}+\left(N_{g}-1\right)}+m \Delta t_{g}} s_{m}\left(t^{*}\right) e^{j 2 \pi f t^{*}} d t^{*} \\
& =P_{m g}(f) S_{m g}(f)
\end{aligned}
$$

where $\Delta t_{g}=\Delta \varphi_{g} /\left(2 \pi f_{T}\right)$. We select the $\Delta t_{g}$ by selecting the range gate limits. This in turn

262 determines $N_{g}$. For a sampling frequency $f_{s}=1 / \Delta t=12 \mathrm{kHz}$, and $\Delta z_{g}=30 \mathrm{~m}$, we get $N_{g}=$

263 1059, and the other range gate parameters shown in Table 1. The time delays are small

264 compared with $\Delta t$, emphasizing the need (at lower sampling frequencies) of using Fourier

265 delays rather than indexing into the time series table. Note that the beam steering time

266 delays are so small that they do not need to be accounted for in the Fourier integral (or

267 indexing for each spectrum). The last column in Table 1 is the velocity increment

268 corresponding to the frequency increment in the Fourier transform. Once these

269 parameters are determined, the $M$ Fourier transforms $S_{m g}\left(f_{i}\right)$ are found for this range gate.

270 Each complex spectral component from $S_{m g}$ is then multiplied by the complex number 
$271 P_{m g}\left(f_{i}\right)$ to form the complex spectrum $S_{m g}{ }_{m g}$. Finally, the $M$ spectra are summed to obtain

272 the spectral components $Q$ of the phased array at range gate $g$.

$$
Q_{g}\left(f_{i}\right)=\sum_{m=1}^{M} P_{m g}\left(f_{i}\right) S_{m g}\left(f_{i}\right)
$$

274 This spectrum is analyzed to find the frequency $f_{T}+\Delta f_{g}$ of the spectral peak, and hence to

275 calculate $\mu_{g}=-c \Delta f_{g} / f_{T}$.

f. Beam sensitivity

277 The overall amplitude response of the phased array is

$$
G \frac{\sin \left[\frac{M}{2} k d\left\{\sin \left(\beta-\beta_{0}\right)-\sin \left(\beta_{g}-\beta_{0}\right)\right\}\right]}{\sin \left[\frac{1}{2} k d\left\{\sin \left(\beta-\beta_{0}\right)-\sin \left(\beta_{g}-\beta_{0}\right)\right\}\right]}
$$

280 where $G$ is the angular sensitivity of an individual microphone at an off-axis angle of $\beta$ -

$281 \beta_{0}$. For the prototype we used Motorola KSN1005A tweeters as microphones, which have

282 an intensity pattern which can be approximated by $\cos ^{5}\left(\beta-\beta_{0}\right)$. The array intensity

283 sensitivity pattern is shown in Figure 10. There are in general two main interference

284 peaks, but the unwanted one of these is pointed well away from the position of the

285 scattered sound, at the time the array is staring at the sensing volume. The $-3 \mathrm{~dB}$

286 beamwidth is $\pm 2^{\circ}$.

The transmitter dish antenna and horn sensitivity have been measured at $3500 \mathrm{~Hz}$

288 (Mikkelsen et al., 2007) with a $-3 \mathrm{~dB}$ beamwidth of $\pm 3^{\circ}$. At $4500 \mathrm{~Hz}$ the beamwidth

289 would be expected to be about $\pm 2^{\circ}$. In the horizontal plane the beamwidth is $\pm 8^{\circ}$, giving

290 reasonable latitude in pointing toward the column being sensed. 
291 3. Hardware design

292 The prototype bi-static system comprises a horn and parabolic dish reflector transmitter,

293 and two identical phased-array receivers. This configuration of a single transmitter which

294 transmits sound vertically, and multiple inclined phased array receivers, is chosen

295 because other configurations, such as a single vertically-pointing receiver and multiple

296 inclined transmitters require more power and the use of multiple transmit frequencies. A

297 master PC generates the transmitted signal, sent to the horn through a power amplifier.

298 The master PC receives signals from one of the phased array receivers (Unit 1), and also

299 generates a trigger signal which is sent to a slave PC. The slave PC controls sampling

300 from a second phased-array receiver (Unit 2). All timing is therefore controlled by the 301 master PC.

302 Each receiver array consists of 12 rows each containing 3 microphones (actually

303 KSN1005A superhorn tweeters used as microphones). The voltage outputs from each

304 group of 3 microphones are summed. This has the effect of confining the lateral

305 (azimuth) receiver sensitivity, while also cancelling some of the random noise. Each of

306 the 12 grouped outputs is amplified, using a low-noise preamplifier, and band-pass

307 filtered. Digitization is achieved using a Data Translation DT9836 usb module, which can

308 sample the 12 channels simultaneously at up to $225 \mathrm{kHz}$ (see Fig. 11). The dish antenna

309 and each receiver are mounted on stand with adjustable zenith angle (see Fig. 12).

310 4. Field test

311 A short field test was conducted to check the basic amplitude and Doppler behavior

312 described above. 
314 The prototype bi-static system was set up at the Riso test facility at Høvsøre, Denmark,

315 with a single transmitter and two phased array receivers. The receivers were each $38 \mathrm{~m}$

316 from the transmitter, with the transmitter-receiver lines at right angles. Receiver Unit 1

317 had hay bales on three sides, as an acoustic shield. Unit 2 and the transmitter had no 318 shielding.

319 The variation of scattered amplitude with height is shown in Fig. 13, using

320 continuous transmission so that the beam steering selectivity could also be tested.

321 Consequently, the large amplitude lobe near the ground comes from the direct signal, but

322 gives an indication that the angular selectivity of the scanning receiver has a half-width of

$32310 \mathrm{~m}$ at the ground, or $15^{\circ}$. However, this apparent beam width is mostly due to the pulse

324 length being equivalent to $8.5 \mathrm{~m}$. The expected profile is also shown, based on Eqs. (6)

325 and (14), and assuming that $T^{2} C_{V}^{2} c^{-2} C_{T}^{-2}$ has a constant value of 50 (see Moulsley et al.,

326 1981, for typical measured values of $C_{V}^{2}$ and $C_{T}^{2}$ ). The unknown overall antenna gain for

327 the expected profile is arbitrarily chosen, but this does not affect the profile shape. In

328 practice the profile results from a convolution, with the sharp nulls in the beam pattern

329 smoothed out.

330 The measured profile closely matches that expected, allowing some confidence in

331 being able to retrieve individual $C_{T}^{2}$ and $C_{V}^{2}$ profiles. To do this, receiver Unit 1 was

332 placed near the transmitter, facing upward. Because $\beta_{1}=90^{\circ}$, only $C_{T}^{2}$ is recorded by

333 Unit 1. Unit 2, still at $38 \mathrm{~m}$ from the receiver and scanning, recorded a combination of 
334 the two structure function parameters. The receiver antennas, while identical, are not

335 calibrated absolutely but, from Eqn. (6)

336

337

$$
\frac{P_{2}}{P_{1}}=2^{23 / 6} \frac{\sin ^{4} \beta}{(1+\sin \beta)^{23 / 6}} e^{-\alpha D(1-\sin \beta) / \cos \beta}\left[1+3.66(1-\sin \beta) \frac{T^{2}}{c^{2}} \frac{C_{V}^{2}}{C_{T}^{2}}\right]
$$

338 allowing the ratio $C_{V}^{2} / C_{T}^{2}$ to be estimated as a function of height $z=D \tan \beta$. Since this

339 experiment does not relate directly to precise wind profiling in complex terrain, the

340 results will be reported elsewhere.

341 b. Scanning bi-static sodar velocity profiles

342 A comparison was available against mast instruments at $44 \mathrm{~m}, 60 \mathrm{~m}$, and $77 \mathrm{~m}$.

343 Fig. 14 shows the mast instrument wind speed record for a three-hours period including a

344 period during which bistatic recordings were being made. Fig. 15 shows the wind speed

345 profile averaged over six 1-second soundings starting at 14:10. For this short run, the

346 error bars are quite large, partly because each spectrum is 1024 points from signals

347 sampled at $12 \mathrm{kHz}$, which gives $85 \mathrm{~ms}$ for the duration of each spectrum and frequency

348 intervals of $12 \mathrm{~Hz}$, equivalent to a velocity interval of nearly $3 \mathrm{~m} \mathrm{~s}^{-1}$. An improved

349 velocity resolution and smaller error bars are obtained by averaging over many more

350 samples.

\section{5. Conclusions}

352 We have described the design and brief field tests of the first scanned bistatic sodar. This

353 new technology potentially has significant advantages over previous bistatic sodars, all of

354 which used a 'staring mode' in which wind data could only be obtained from a confined 
355 height range. The main motivation for designing a scanning bistatic sodar, described in

356 the first section, is to avoid errors arising in all current sodars and lidars when they

357 sample non-horizontally-uniform winds. This situation arises generically in complex

358 terrain and, without a solution such as the new bistatic sodar, wind estimates in such

359 regions are considerably compromised.

360 The result is single-column, or 'mast-like' sampling of the wind profile. But there

361 are other advantages which we have identified. These include

362 - improved SNR because of the extra scattering from velocity fluctuations

363 - much improved performance in neutral lapse conditions, where the turbulent

364 temperature fluctuation contrast is low

365 - improved rejection of rain echoes through an advantageous scattering pattern

366 - larger Doppler shift reducing the possibility of erroneous velocity estimates

367 arising from echoes from fixed structures

368 We describe the relevant theory for each of these factors, and how to design a

369 scanning sodar which has good spatial resolution. In particular, it is important to use a

370 pulsed system to avoid the multiple overlapping spectra experienced by the Heimdall

371 sodar (Mikkelsen, 2007). In fact, the pulse length largely determines the vertical

372 resolution in the scanned bistatic system. The spectral processing needs to be done rather

373 carefully, and certainly is rather more complicated than for a monostatic system.

374 Nevertheless, we found all spectral processing, and post-sampling beam steering, can

375 readily be completed in MATLAB in a small fraction of the profiling time, and

376 effectively gives real-time performance. 
A prototype scanning bistatic sodar was designed using a dish antenna transmitter

378 and $12 \times 3$ arrays of microphones for the receivers. The baseline used in our experiments

379 was $38 \mathrm{~m}$, but this is somewhat arbitrary and there should be further exploration of the

380 optimum configuration. No acoustic baffles (except for crude use of some hay bales)

381 were used in our prototype. We would expect significant improvements in performance

382 if properly-designed acoustic shielding was used.

383 Very preliminary experiments are described. The profile of the turbulent

384 scattering intensity is found to closely approximate what we expect from theory, giving

385 some confidence in the instrument design and scanning. Comparisons were performed

386 against mast-mounted instruments, and the velocity profile obtained with the bistatic

387 sodar agreed with the 'standard' instruments to within measurement uncertainties.

388 We are now progressing to designing microphone-based arrays as an optional

389 addition to a monostatic sodar. This configuration will allow both monostatic and bistatic

390 configuration to operate simultaneously, or sequentially, thereby providing considerable

391 self-checking of the instrument, since the two velocity estimation schemes are quite

392 different.

$393 \quad$ Acknowledgements

394 This work was supported by funding from the European Commission under WP6 of

395 project Upwind. The authors are grateful for help by staff of Riso -DTU, and in

396 particular Mike Courtney and the Høvsøre field team led by Bjarne.

\section{References}

398 AQSystem http://www.aqs.se/ 
399 Behrens, P., J. O'Sullivan, R. Archer, and S. Bradley, 2012: Underestimation of mono-

400 static sodar measurements in complex terrain. Bound. Layer Met., 143, 97-106.

401 Bingol, F., J. Mann, and D. Foussekis, 2009: Conically scanning lidar error in complex

402 terrain. Meteorologische Zeitschrift, 18, 189-195.

403 Bradley, S. G., 1999: Use of coded waveforms for SODAR systems, Met. Appl. Phys. 71,

$404 \quad 15-22$.

405 Bradley, S. G., 2007: Atmospheric Acoustic Remote Sensing. Florida, CRC Press/

406 Taylor and Francis Group, 328pp.. ISBN 978-0-8493-3588-4.

407 Bradley, S. G., 2008: Wind speed errors for LIDARs and SODARs in complex terrain.

408 IOP Conf. Series: Earth and Environmental Science, Roskilde, Denmark, 1, 1-7.

409 Bradley, S. G. and T. Webb, 2002: Use of an Ultrasonic Sodar to Sense Raindrop Size

410 Distributions. J. Atmos. Ocean. Tech., 19, 1203-1207.

411 Bradley, S. G., Y. Perrott, and A. Oldroyd, 2012: Corrections for Wind-Speed Errors

412 from Sodar and Lidar in Complex Terrain. Bound. Layer Met, 143, 37-48.

413 Coulter, R. L. and K. H. Underwood, 1980: Some Turbulence and Diffusion Parameter

414 Estimates within Cooling Tower Plumes Derived from Sodar Data. J. Appl. Meteor., 19, $415 \quad 1395-1404$.

416 Emeis, S., 2010: Surface-based Remote Sensing of the Atmospheric Boundary Layer.

417 ISBN-10 9048193397, Atmospheric and Oceanographic Sciences Library, Springer-

$418 \quad$ Verlag, pp 174.

419 Mastrantonio, G., G. Fiocco and A. Marzorati, 1986: Simultaneous operation of a sodar 420 system in monostatic and bistatic modes: Preliminary results. Atm. Res., 20, 213-223. 
421 Mathews, T., D. A. Bohlender and D. Smith, 1986: Effect of refraction of sound on

422 bistatic doppler sodar measurements of wind profiles. Atm. Res., 20, 141-149.

423 Mikkelsen, T., 2007: The Bistatic Sodar "Heimdall" - You blow, I listen, Riso National

424 Laboratory DTU, ISBN 87-550-3250-8

425 Moulsley, T. J. and R. S. Cole, 1993: A general radar equation for the bistatic acoustic

426 sounder. Bound. Layer Met., 19, 359-372.

427 Moulsley, T.J., D.N. Asimakopoulos, R.S. Cole, B.A. Crease and S.J. Caughey, 1981:

428 Measurement of boundary layer structure parameter profile by using acoustic sounding

429 and comparison with direct measurements. Quart. J. Roy. Meteor. Soc., 107, 203-230.

430 Natural Power ZephIR Lidar. http://www.naturalpower.com/zephir-300-wind-lidar

431 Shamanaev, S. V., 2003: Acoustic Sounding of Raindrop Size Distribution. J. Atmos.

432 Ocean. Tech. 20, 152-158.

433 Thomson, D. W. and R. Coulter, 1974: Analysis and Simulation of Phase Coherent

434 Acdar Sounding Measurements, J. Geophys. Res., 79, 5541-5549.

435 Underwoord, K. H., 1981: Sodar signal processing methods and the Riso-78 experiment.

$436 \mathrm{PhD}$ dissertation. Pennsylvania State University, 178pp.

437 Upwind EU Project, 2011: http://www.upwind.eu/Paginas/Work packages/6 Remote

438 sensing.aspx

439 Wesely, M. L., 1976: The combined effect of temperature and humidity fluctuations on

440 refractive index. J Appl Met. 15, 43-49.

441 Zinichev, V. A., N. A. Mityakov, V. O. Rapoport, and Yu A. Sazonov, 1997: Bistatic

442 sodar based on 15-meter overall review radiotelescopes. Radiophys Quant Opt., 40, 877-

$443 \quad 878$

444 
445 TABLE 1 . Typical parameters for $f_{s}=12 \mathrm{kHz}, D=50 \mathrm{~m}, z_{0}=60 \mathrm{~m}$, and a design vertical 446 resolution of $20 \mathrm{~m}$.

\begin{tabular}{|c|c|c|c|}
\hline$z_{g}[\mathrm{~m}]$ & $\Delta t_{g}[\mu \mathrm{s}]$ & $i_{g}$ & $\Delta u\left[\mathrm{~m} \mathrm{~s}^{-1}\right]$ \\
\hline 20 & -125 & 353 & 1.0 \\
\hline 50 & -21 & 1412 & 1.4 \\
\hline 80 & 30 & 2471 & 2.0 \\
\hline 110 & 58 & 3529 & 2.6 \\
\hline 140 & 74 & 4588 & 3.3 \\
\hline 170 & 85 & 5647 & 3.9 \\
\hline 200 & 92 & 6706 & 4.6 \\
\hline 160 & 82 & 5294 & 3.7 \\
\hline 180 & 88 & 6000 & 4.1 \\
\hline 200 & 92 & 6706 & 4.6 \\
\hline
\end{tabular}

447

448

449 


\section{$450 \quad$ List of Figures}

451 FIG. 1. The bi-static geometry for transmission in direction $\mathbf{T}$ and reception from

452 direction $\mathbf{R}$

453 FIG. 2. Dual bi-static geometry of Risø-78.

454 FIG. 3. Comparison between mast (circles) and bi-static sodar measurements (triangles)

455 from the Ris $\varnothing-78$ experiment (adapted from Underwood, 1981).

456 FIG. 4. Heimdall bi-static spectrum (adapted from Mikkelsen et al., 2007)

457 FIG. 5. The geometry of the basic column-profiling bi-static sodar.

458 FIG. 6. Relative scattering contributions from turbulent temperature fluctuations (long

459 dashes) and turbulent velocity fluctuations (solid line), versus height, for $D=50 \mathrm{~m}$. Also

460 shown (short dashes) is the velocity fluctuation response for $D=30 \mathrm{~m}$. The dotted line is

461 the response for a mono-static sodar. Also shown (circles) is the response from rain, with

462 arbitrary scaling.

463 FIG. 7. Sensitivity to spectral corruption due to echoes from fixed objects. Typical sodar

464 a parameters are used, as described in the text, and the hard reflecting surface is at a

465 range of $20 \mathrm{~m}$. Combined spectra from the hard surface and the atmosphere are shown for

466 the mono-static case (solid line) and the bi-static case (dotted line).

467 FIG. 8. The three Gaussian spatial functions defining the bi-static sampling volume.

468 FIG. 9. Sampling volume sensitivity, relative to 1 at the centre, for pointing heights of 30,

46950 , and $80 \mathrm{~m}$. Parameters are $f_{\mathrm{T}}=4500 \mathrm{~Hz}, L=0.8 \mathrm{~m}, D=50 \mathrm{~m}$, and pulse sigma $=0.02 \mathrm{~s}$.

470 FIG. 10. Array sensitivity [dB] for no phase shift (solid line) and $30^{\circ}$ shift in pointing

471 angle (dashed line).

472 FIG. 11. The hardware system for the prototype bi-static sodar. 
473 FIG. 12. The dish antenna transmitter and one of the phased array receivers.

474 FIG. 13. The variation of received signal amplitude with height. Measurements (solid

475 line), and modelled (dashed line).

476 FIG. 14. Wind speed recorded on mast instruments at $44 \mathrm{~m}$ (solid), $60 \mathrm{~m}$ (short dashes), 477 and $77 \mathrm{~m}$ (long dashes)

478 FIG. 15. Wind speeds from the bistatic sodar (solid line and dots) compared with wind 479 speeds from mast instruments (crosses).

480 
Click here to download Non-Rendered Figure: JTECH-D-11-00035_Bradley_Fig1c.doc 


\section{Fig 2}

Click here to download Non-Rendered Figure: JTECH-D-11-00035_Bradley_Fig2c.doc 


\section{Fig 3}

Click here to download Non-Rendered Figure: JTECH-D-11-00035_Bradley_Fig3c.doc 
Click here to download Non-Rendered Figure: JTECH-D-11-00035_Bradley_Fig4c.doc 


\section{Fig 5}

Click here to download Non-Rendered Figure: JTECH-D-11-00035_Bradley_Fig5c.doc 
Fig 6
Click here to download Non-Rendered Figure: JTECH-D-11-00035_Bradley_Fig6c.doc

${ }^{3}$ Click here to down

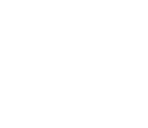

$\sqrt{3}$

$\sqrt{2}$ (1) (1) (1) (1) $\sqrt{2}$ .

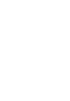
更

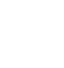

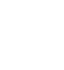

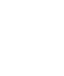

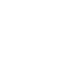

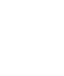

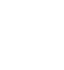

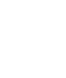
更

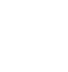

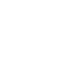

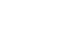


Fig 7
Click here to download Non-Rendered Figure: JTECH-D-11-00035_Bradley_Fig7c.doc 
Fig 8
Click here to download Non-Rendered Figure: JTECH-D-11-00035_Bradley_Fig8c.doc 
Click here to download Non-Rendered Figure: JTECH-D-11-00035_Bradley_Fig9c.doc 
Click here to download Non-Rendered Figure: JTECH-D-11-00035_Bradley_Fig10c.doc 
Fig 11
Click here to download Non-Rendered Figure: JTECH-D-11-00035_Bradley_Fig11c.doc

(

here to download Non-Rendered Figure: JTECH-D-11-00035_Bradley_Fig11c.doc

(2)

(2)

Click here to download 
Click here to download Non-Rendered Figure: JTECH-D-11-00035_Bradley_Fig12c.doc 
Fig 13
Click here to download Non-Rendered Figure: JTECH-D-11-00035_Bradley_Fig13c.doc

Fig 13
Click here to download Non-Rendered Figure: JTECH-D-11-00035_Bradley_Fig13c.doc 
Fig 14
Click here to download Non-Rendered Figure: JTECH-D-11-00035_Bradley_Fig14c.doc 
Click here to download Non-Rendered Figure: JTECH-D-11-00035_Bradley_Fig15c.doc 